\title{
Study on the performance of the Particle Identification Detectors at LHCb after the LHC First Long Shutdown
}

\author{
Marianna Fontana*广 \\ CERN and INFN \\ E-mail: marianna.fontana@cern.ch
}

\begin{abstract}
During the First Long Shutdown (LS1), the LHCb experiment has introduced major modification in the data-processing procedure and modified part of the detector to deal with the increased energy and the increased heavy-hadron production cross-section. In this contribution we review the performance of the particle identification detectors at LHCb, Rich, Calorimeters, and Muon system, after the LS1.
\end{abstract}

38th International Conference on High Energy Physics 3-10 August 2016

Chicago, USA

* Speaker.

${ }^{\dagger}$ On behalf of the LHCb collaboration. 


\section{Introduction}

A vast sample of physics analyses in LHCb rely on Particle Identification (PID) variables to identify and separate different types of charged particles (muons, pions, kaons, protons and electrons). The performance of these variables as selection criteria could in principle be measured using simulated samples. However this is not the optimal procedure as the PID informations are not reproduced in Monte Carlo (MC) samples at the level of needed accuracy for the analysis. At this scope a data-driven technique has been developed, which makes use of calibration samples, selected without the use of PID informations from the RICH, CALO and MUON systems.

\section{The LHCb experiment and the PID subdetectors}

The LHCb detector [3] is a single-arm forward spectrometer covering the pseudorapidity range $2<\eta<5$. The detector includes a high-precision tracking system consisting of a silicon-strip vertex detector surrounding the $p p$ interaction region, a large-area silicon-strip detector located upstream of a dipole magnet, and three stations of silicon-strip detectors and straw drift tubes placed downstream of the magnet. The identification of different type of particles is done with three different sub-systems, described in the following in more details.

Different types of charged hadrons are distinguished, over the momentum range $2-100 \mathrm{GeV} / \mathrm{c}$, using information from two ring-imaging Cherenkov detectors [1]. The RICH 1 is located upstream of the dipole magnet and uses $C_{4} F_{10}$ gas as radiator. In Run 1 a second radiator (aerogel) was present but found to be of reduced effectiveness and for this reason removed for Run 2. The RICH 2 is located downstream of the magnet and has a $C F_{4}$ radiator. It is responsible for the high momentum coverage.

Muons are identified by a system consisting of five stations [2]: the first one (M1) is located in front of the calorimeter and used for the measurement of the transverse momentum at the hardware trigger level. The other stations (M2-M5) are placed behind the hadronic calorimeter and are interspersed with iron walls used as muon filters. The stations make use of the Multi wire Proportional Chambers (MWPCs) technology, except for the inner region of M1 where Gas Electron Multiplier (GEM) detectors are used. The stations are divided into four regions, each one equipped with chambers of different granularities to cope with the large variation in particle rates from the central to the peripheral part of the detector.

Photons, electrons and hadrons are identified thanks to the information provided by a four detectors system: scintillating-pad (SPD) and preshower (PS) detectors, an electromagnetic calorimeter (ECAL) and a hadronic calorimeter (HCAL). The SPD and the PS both consist of a plane of scintillator tiles, separated from each other by a thin lead layer, while the ECAL and HCAL have shashlik and sampling constructions, respectively.

The information obtained separately by each PID subdetectors can be combined to obtain more discriminating variables. The likelihood information produced by each sub-system is added linearly, to form a set of combined likelihoods (DLL). These variables give a measure of how likely the mass hypothesis under consideration is, for any given track, relative to the pion hypothesis, e.g. for kaons $D L L(K-\pi)$. A second approach makes use of multivariate discriminant to combine the inputs from the PID subdetectors in addition to other tracking information. 
The online event selection is performed by a trigger, which consists of a hardware stage (L0), based on information from the calorimeter and muon systems, followed by a two-steps software stage (HLT1-HLT2), which applies a full event reconstruction.

\section{The PID calibration samples}

During Run 1, despite the size of the calibration samples, some analysis faced the problem of being dominated by systematic uncertainty related to the PID selection efficiency, especially in those regions of phase-space with low-statistics. For Run 2 a new strategy has been chosen in order to face this problem and keep systematics effects under control [6,7]. First the selections have been optimised to enhance the purity and the kinematic coverage of the samples. Secondly the samples are selected directly at the trigger level. In fact the PID variables in Run 2 are used in physics lines at the HLT2 level of the software trigger, which may introduce differences with respect to the same variables calculated with the offline reconstruction. It is essential to ensure the possibility of measuring the performance for both of them. A very important feature is that L0 and Hlt1 trigger do not introduce any bias to the PID distributions of the calibration samples.

The HLT2 selections are designed to select pure samples of low-multiplicity decay modes with large branching fractions. An overview of decay modes is given in Table 3. The decays used to obtain muon and electron calibration samples, are selected with the tag-and-probe method, where PID requirements are made only on one of the two tracks, while the other is used to obtain the performance. Other modes, like the ones for pions and kaons, instead are selected using kinematical information only. More than one sample is selected for each species to maximise the kinematic coverage.

\begin{tabular}{lll} 
Species & Low $p-p_{\mathrm{T}}$ & High $p$ and $p_{\mathrm{T}}$ \\
\hline$e^{ \pm}$ & \multicolumn{1}{c}{-} & $J / \psi \rightarrow e^{+} e^{-}$ \\
$\mu^{ \pm}$ & $D_{s}^{+} \rightarrow \mu^{+} \mu^{-} \pi^{+}$ & $J / \psi \rightarrow \mu^{+} \mu^{-}$ \\
$\pi^{ \pm}$ & $K_{\mathrm{S}}^{0} \rightarrow \pi^{+} \pi^{-}$ & $D^{*} \rightarrow D^{0}\left(K^{-} \pi^{+}\right) \pi^{+}$ \\
$K^{ \pm}$ & $D_{s}^{+} \rightarrow K^{+} K^{-} \pi^{+}$ & $D^{*} \rightarrow D^{0}\left(K^{-} \pi^{+}\right) \pi^{+}$ \\
$p^{ \pm}$ & $\Lambda^{0} \rightarrow p \pi^{-}$ & $\Lambda^{0} \rightarrow p \pi^{-}, \Lambda_{c}^{+} \rightarrow p K^{-} \pi^{+}$
\end{tabular}

Table 1: Overview of decay modes that are used to select calibration samples.

The PID distributions for the the signal only are obtained by subtracting the background statistically with the $s$ Plot method [5], using the invariant mass distribution as discriminating variable. Examples of mass distributions for some of the lines are shown in Fig. 1, together with the fit functions.

\section{The performance}

The performance for each particle have been determined using the main samples produced during 2015. The kaon performance are studied using the $D^{*+} \rightarrow D^{0} \pi^{+}$decay, reconstruct purely from kinematic selections. The kaon efficiency and the pion to kaon misidentification rate as a 

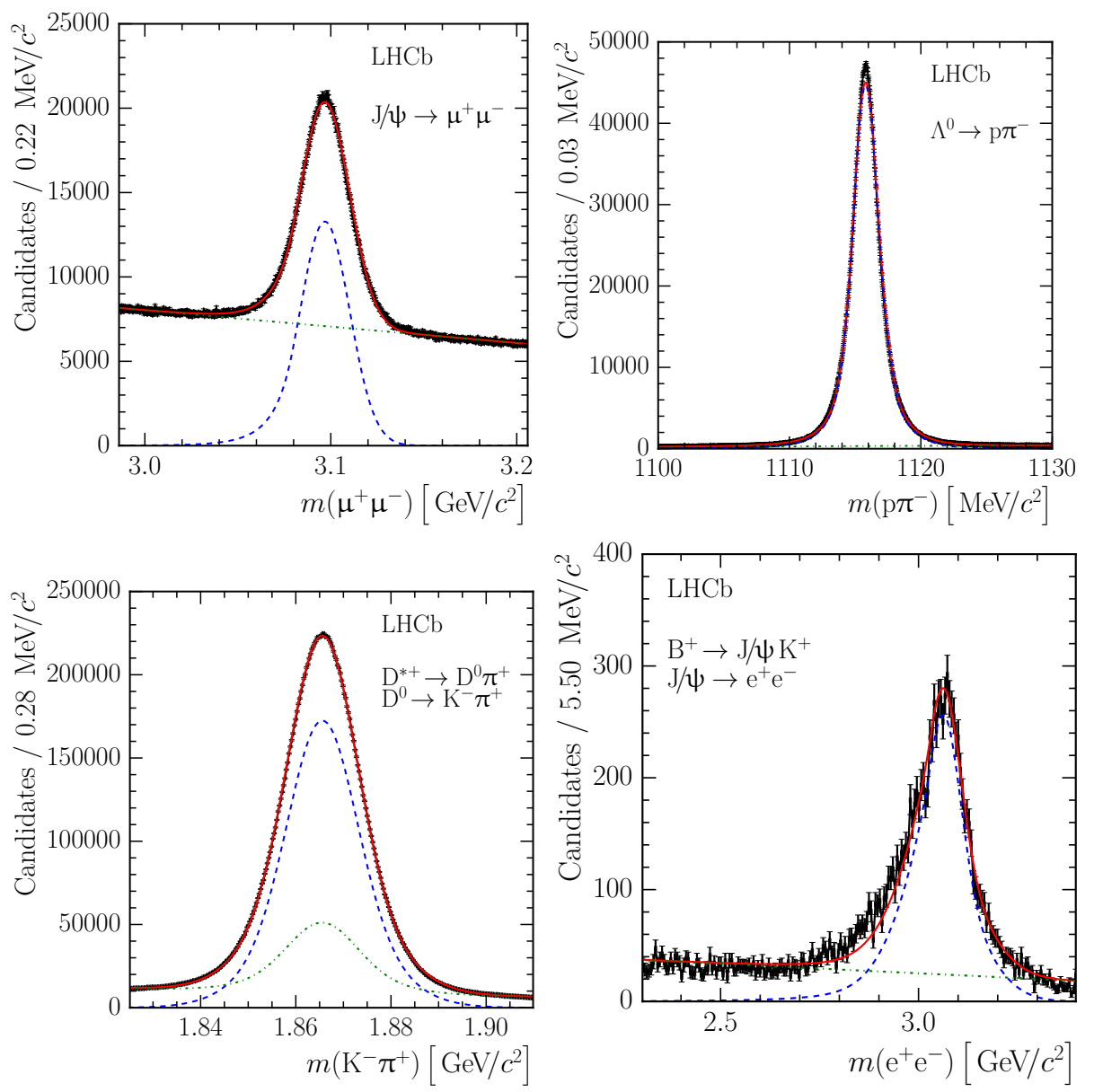

Figure 1: Invariant mass distribution of the $J \psi \rightarrow \mu^{+} \mu^{-}$(top left), $\Lambda^{0} \rightarrow p \pi^{-}$(top right), $D^{0} K^{-} \pi^{+}$(bottom left) and $J / \psi \rightarrow e^{+} e^{-}$(bottom right) candidates with the fit function overlaid. Data are represented as black dots, while the total fit function is the red line. The blue dashed line is the signal fit component and the green dotted line is the background fit component.

function of the momentum are reported in Fig. 2, where also the Run 1 performance are presented for comparison. It is worth to note that while the kaon efficiency remains the same, the misidentification probability profits of the aerogel removal and gives better performance at low momentum. When averaging over the momentum range the kaon efficiency is about $95 \%$ with a pion misidentification rate of $10 \%$. With a more stringent requirement on the $D L L$ variable the misidentification is reduced to $3 \%$ for a loss of only $10 \%$ on the identification.

The performance of the muon identification is obtained from data using muons from $J \psi \rightarrow$ $\mu^{+} \mu^{-}$decays and pions from $D^{0} \rightarrow K^{-} \pi^{+}$decays. The muon identification requirement is based on the association of hits around the extrapolated trajectory of a track in the muon system (isMuon). The background rejection power can then be improved by making a selection on the $D L L$ distribution. Figure 3 shows, as a function of the track momentum the efficiency of the muon candidate selection, and the probabilities of incorrect identification of protons, pions and kaons as muons.

The electron identification performance is evaluated using the $B^{ \pm} \rightarrow J / \psi\left(e^{+} e^{-}\right) K^{ \pm}$decay 

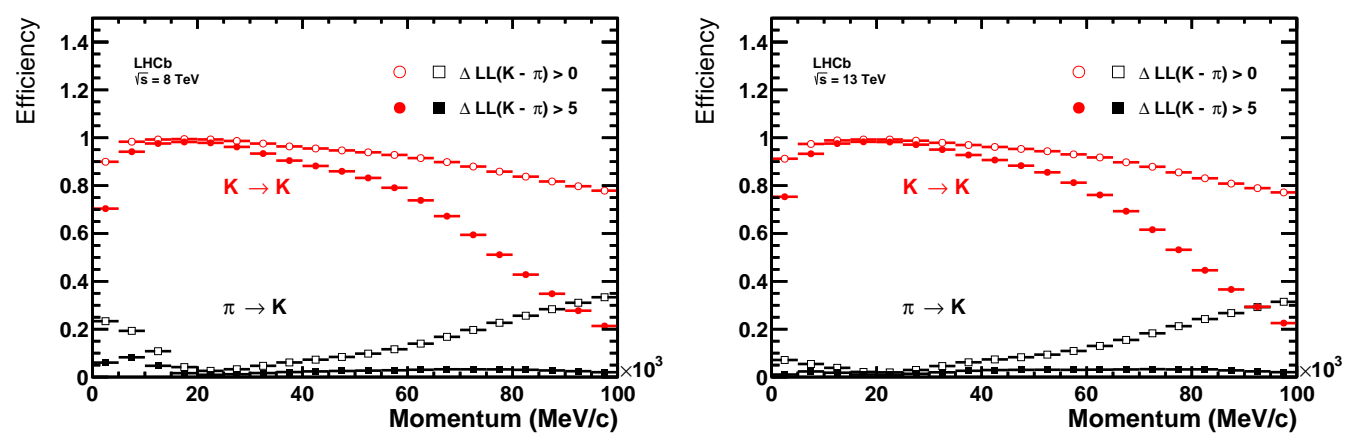

Figure 2: Kaon identification efficiency and pion misidentification rate as measured using 2012 (left) and 2015 (right) data as a function of track momentum. Two different $\Delta L L(K-\pi)$ requirements have been imposed on the samples, resulting in the open and filled marker distributions, respectively.
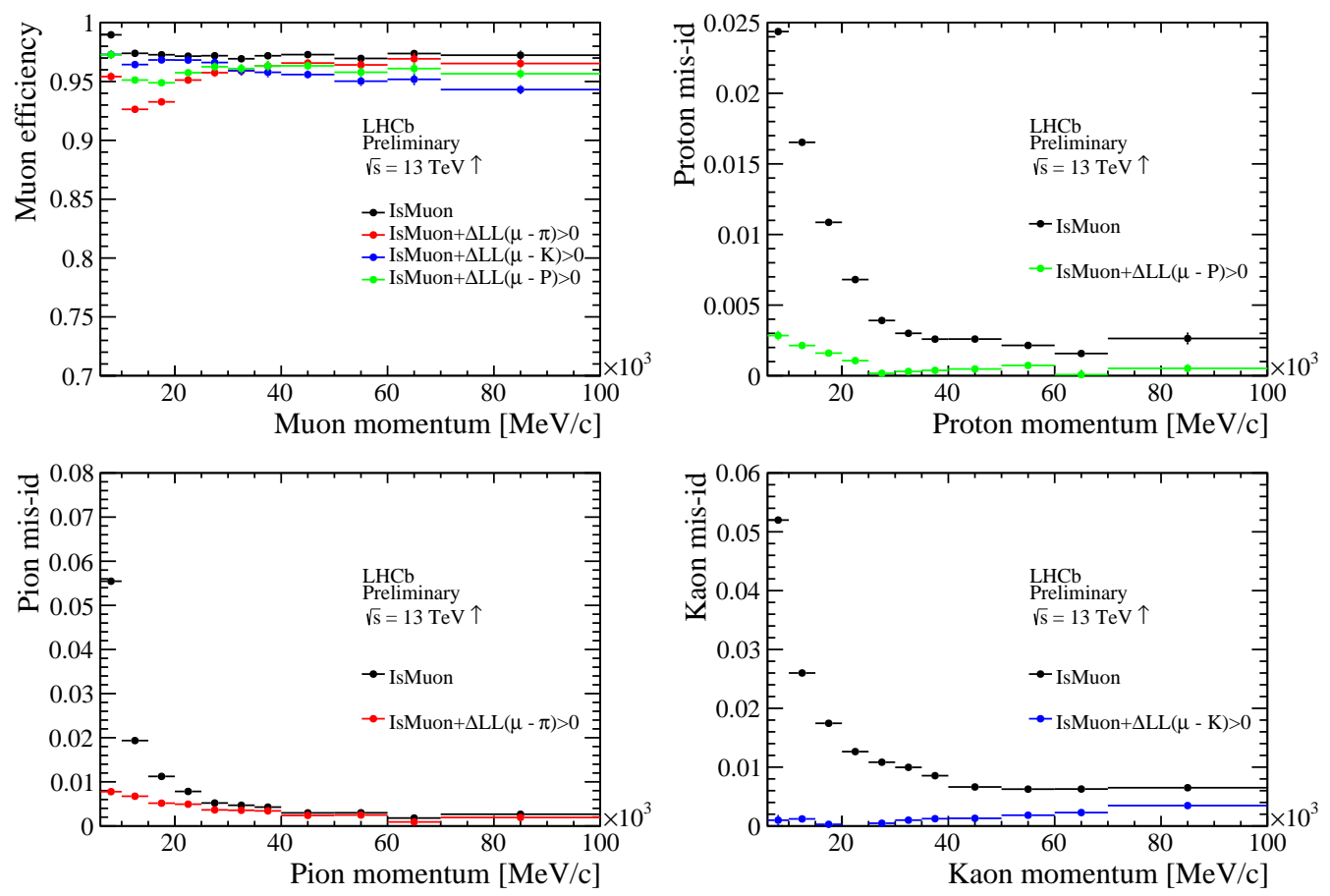

Figure 3: (Top left) Efficiency of the isMuon selection as a function of momentum (black) and efficiency of the isMuon selection plus $\Delta L L(\mu-h)>0$, where $h$ can be a pion (red), a kaon (blue) and a proton (green). (Top right) Misidentification probability of pions as a function of momentum for isMuon (black) and isMuon $+\Delta L L(\mu-\pi)>0$ (red). (Bottom left) Misidentification probability of kaons as a function of momentum for isMuon (black) and isMuon $+\Delta L L(\mu-K)>0$ (blue). (Bottom right) Misidentification probability of protons as a function of momentum for isMuon (black) and isMuon $+\Delta L L(\mu-P)>0$ (green). The sample is from 2015 data taking, with the up polarity of the magnet (up arrow). 
with a tag-and-probe method, where one of the electrons is required to be identified by its electron ID while the second electron is selected without using any information from the calorimeter system. This second electron is then used to estimate the efficiency of the electron ID, shown in Fig. 4 for Run 1 data [4]. The average identification efficiency of electrons from the events is $\sim 90 \%$ for a misidentification rate of $\sim 4 \%$. The performance are expected to be the same also for Run 2 .

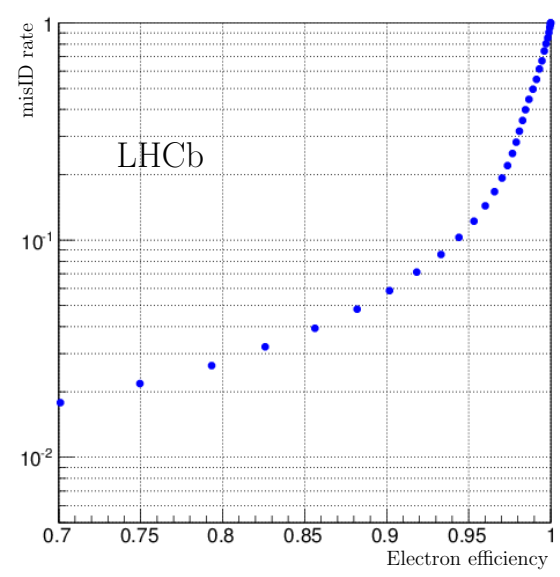

Figure 4: Electron identification efficiency versus hadron misidentification rate.

\section{Conclusion}

During the LS1 only slight modifications have been implemented on PID subdetectors. However to cope with the more stringent requests for Run 2 a new procedure has been introduced to select the PID calibration samples directly at trigger level. The selection of the samples have improved the purity, leading to lower statistical uncertainties and better performance. The improvements open the door to a large number of PID-related studies, which will result in a better understanding of the systematic effects related to the detector. All these features pave also the way for an improved PID performance for the LHCb upgrade.

\section{References}

[1] M. Adinolfi et al. [LHCb collaboration], Eur. Phys. J. C 73 (2013) 2431.

[2] A. Alves et al. [LHCb collaboration] J. Instrum. 8 (2013) P02022.

[3] A. Alves et al [LHCb collaboration] J. Instrum. 3 (2008) S08005.

[4] R. Aaij et al. [LHCb collaboration] Int.J.Mod.Phys. A30 (2015) 1530022.

[5] M. Pivk and F. R. Le Diberder, 720 Nucl. Instrum. Meth. A555 (2005) 356, arXiv:physics/0402083.

[6] L. Anderlini, S. Benson, O. Lupton, B. Sciascia, V. Gligorov, LHCb-PUB-2016-020.

[7] L. Anderlini, S. Benson, O. Lupton, B. Sciascia, V. Gligorov, LHCb-PUB-2016-005. 